\title{
La medicina fra intelligenza artificiale e necessità di un nuovo Umanesimo
}

\author{
Antonio Barracca ${ }^{1}$, Stefano Ledda², Gianmaria Mancosu ${ }^{3}$, Walter Melis ${ }^{4}$, Maria Rosa Rinaldi ${ }^{5}$, Patrizia Francesca Patricelli ${ }^{6}$ \\ ${ }^{1}$ Medico, specialista in Nefrologia e Urologia, esperto di applicazioni per medici su piattaforma Android e Apple, Cagliari \\ ${ }^{2}$ Ingegnere Elettronico, Sviluppatore di App, Cagliari \\ ${ }^{3}$ Ingegnere Elettronico, Dottore di ricerca in Informatica, Sviluppatore di App, Cagliari \\ ${ }^{4}$ Data Analyst, Cagliari \\ ${ }^{5}$ Esperta in Web e Digital Marketing in Sanità, Perugia \\ ${ }^{6}$ Medico, specialista in Nefrologia, Centro Dialisi, Ospedale SS. Trinità, ASSL Cagliari, Cagliari
}

\begin{abstract}
A new humanism for medicine in the digital age
The digital revolution, computer science and artificial intelligence are pervading all aspects of our lives. The world of health is no exception, starting with the digitization of images, through the collection of data from major clinical trials, up to the building of Big Data that contain the clinical histories of millions of patients. Then came cognitive computers and machine learning. Artificial intelligence has begun to occupy spaces previously occupied by doctors, with automatic reporting of radiographic images, support for diagnosis, quick database consultation with decision-making algorithms, and many other applications. At the same time, the reliability of these systems has improved. Like any change that alters old balances, this revolution must also be governed by the redefining of roles and priorities. The need is emerging for a new humanism that redefines the role of doctors in their relationship with patients. It will give patients the certainty that artificial intelligence, so important to health, will always remain within human decisions.
\end{abstract}

Keywords: Precision medicine, Artificial intelligence, Cognitive computing, New humanism

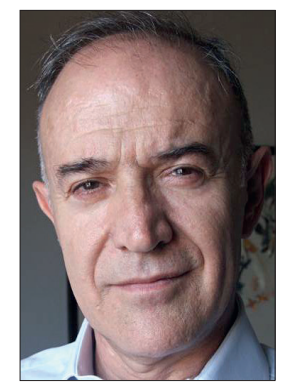

Arthur C. Clarke autore del libro (1) e Stanley Kubrick, regista di 2001: Odissea nello spazio (2), nel lontano $1968 \mathrm{ci}$ mostrarono un mondo immaginario nel quale un super computer con intelligenza artificiale, capace di riprodurre tutte le attività della mente umana, provare emozioni, parlare con una voce totalmente umana, ma dal timbro freddo e asettico che ben rendeva l'idea di un'intelligenza artificiale, dialogando con gli astronauti, governava l'astronave "Discovery 1 " in ogni singolo aspetto della missione spaziale. II super computer si chiamava HAL 9000.

Accepted: June 29, 2017

Published online: July 19, 2017

Indirizzo per la corrispondenza:

Dr. Antonio Barracca

Medico, Specialista in Nefrologia e Urologia

Viale Merello, 74

09123 Cagliari

barraccaantonio@gmail.com
II suo nome derivava ufficialmente dalle lettere " $H$ "euristic e " $A L$ "gorithmic (3). La scelta ovviamente non fu casuale. Infatti mentre un algoritmo è una sequenza definita da eseguire per la risoluzione di un dato problema, tale per cui dagli stessi input si ottengono in maniera deterministica gli stessi risultati, l'approccio euristico non segue un percorso chiaro, ma si affida all'intuito e allo stato temporaneo delle circostanze, al fine di generare nuova conoscenza. Dunque HAL 9000 conteneva già nel proprio nome quello che sarà il suo destino, il conflitto tra intuito e ragionamento, che lo porterà a tentare di sterminare l'equipaggio dell'astronave (4).

\section{L'intelligenza artificiale}

Dopo quarantotto anni la fantasia di Arthur C. Clarke ha assunto la concretezza del valore scientifico per cui la strada è stata aperta. L'intelligenza artificiale (AI) ha ora una propria validità scientifica, metodologia di ricerca, strumenti informatici validi a supportare l'enorme quantità di informazioni che devono essere elaborate perché i campi di applicazione si sono dilatati. Ross (5) è il primo avvocato digitale. È basato sulla tecnologia Watson, il primo computer cognitivo di IBM (6). Si occupa di bancarotta nello studio Baker \& Ho- 
stetler negli Stati Uniti. Sa leggere, comprende il linguaggio degli avvocati, sa formulare ipotesi, fare ricerche e trovare soluzioni. È infaticabile. II suo costo mensile è pari al compenso orario di un avvocato. La JP Morgan Chase Bank si occupa di servizi finanziari, ha 90 milioni di clienti e 265 mila impiegati, 15 mila sono ingegneri, si occupano di automazione e intelligenza artificiale applicata ai servizi finanziari. Qualsiasi domanda di finanziamento bancario è gestita direttamente da un computer cognitivo (7). Questo prende in carico la richiesta di finanziamento, ne valuta la congruità, le capacità finanziarie del richiedente, i rischi di esposizione della banca e decide se concedere o no il prestito bancario.

Siamo di fronte a un capovolgimento del nostro punto di vista verso le macchine, i robot. È vero che finora si è pensato a come insegnare qualcosa ai robot. II progresso della conoscenza ha invertito il problema. Stiamo costruendo macchine che imparano da sole quello che devono fare. Machine Learning (8) questo è il nome di un campo della computer science che dà ai computer la capacità di imparare senza essere stati esplicitamente programmati. Si chiama Deep Learning (DL), ossia apprendimento "profondo".

\section{Il computer che sa giocare a poker}

Si chiama Libratus (10), e ha partecipato al torneo nella seconda edizione di The Brains vs Artificial Intelligence. $\grave{E}$ stata I'Alberta Machine Intelligence Institute (Amii) in Canada nel 2015 a produrre il primo campione di poker sintetico, DeepStack (11). Perché si sta sperimentando così tanto con i giochi? Il poker è importante perché quando si gioca a scacchi, quello che l'avversario sta pensando non è rilevante, è rilevante quello che c'è sulla scacchiera. La scacchiera contiene già tutte le possibili variabili del gioco. Con il poker le informazioni sul tavolo sono molte meno e il comportamento dei giocatori è una delle variabili fondamentali difficili da prevedere. Ma aver battuto a Pittsburgh (USA) quattro dei giocatori più forti al mondo rappresenta la prima soluzione di quello che in teoria dei giochi viene definito "gioco a informazione imperfetta". Nei giochi a informazione perfetta, come scacchi e dama, ogni giocatore conosce in ogni momento la storia delle giocate precedenti. Nel poker o nella briscola, classificati appunto a informazione imperfetta, ogni giocatore deve prendere di volta in volta una decisione senza sapere quali carte hanno in mano gli altri (12). II risultato ottenuto da Libratus è quindi importante perché, per la prima volta, è stato possibile legare la teoria dei giochi alla teoria delle decisioni. L'aspetto più sorprendente è che nessuno ha insegnato a Libratus come giocare a poker. Gli sono state date le regole e Libratus ha cominciato a giocare milioni di partite e ha imparato per conto suo, per un equivalente di circa 2.900 anni di calcolo nel Pittsburgh Supercomputing Center.

\section{Dr. Watson, un medico che sa tutto}

Ma la sfida di Watson Health è più insidiosa. È vero, può leggere 200 milioni di pagine di testo in 3 secondi, ma può essere "allenato". Le sue competenze oncologiche sono state sviluppate al Memorial Sloan Kettering Cancer Center di New York (USA) (13). Watson usa lo stesso modello di ragionamento probabilistico che il medico adotta per arrivare a una diagnosi ed è capace di apprendere dalle evidenze scientifiche. Se gli vengono esposti i sintomi di un paziente seleziona quelli più significativi, ne analizza la storia clinica, esamina linee guida e database e formula ipotesi per fornire raccomandazioni classificate per livello di evidenza. Può suggerire gli esami necessari per affinare la diagnosi. Per ora è usato come supporto ai medici nelle decisioni cliniche, come tutor cognitivo per gli studenti di medicina (14). Ma sa fare molto altro.

Ma per proseguire abbiamo bisogno di delimitare il campo della intelligenza artificiale. Noi medici siamo di fronte a una massa di dati che provengono da esami di laboratorio, sensori elettronici, cartelle cliniche. Una miniera enorme di informazioni sanitarie che messe in ordine ci possono aiutare a delineare ogni fase del procedimento clinico dall'anamnesi, alla diagnosi e alla terapia. È quanto fa Watson Health scavando nelle banche dati alla ricerca di modelli, per mettere insieme storie cliniche simili, confrontando esami di laboratorio e dati clinici e cercando i dati della letteratura. Tutto questo lavoro fornisce ai medici previsioni, suggerimenti, elementi di supporto che aiutano il medico a prendere decisioni più appropriate. Nelle prime fasi i computer vengono addestrati a imparare regole di "buon senso" e "I'istinto del medico". Di recente, ad esempio, è stata valutata la perizia di Watson in campo oncologico. Watson Health ha esaminato 638 cartelle cliniche di pazienti con cancro al seno e per ciascuna paziente ha generato una prescrizione medica personalizzata in un tempo medico di 40 secondi, contro i 12 minuti di un medico. Le prescrizioni in questo tipo di patologia senza metastasi sono state in circa l' $80 \%$ dei casi in accordo con quelle formulate dagli oncologi del Manipal Comprehensive Cancer Center (Bangalore, India).

Facciamo un altro passo avanti per delineare uno scenario che occuperà e già occupa i nostri ospedali e diventerà la norma. Questo riguarda uno spicchio dell'attività clinica che avrà anche un forte impatto sul ruolo del medico. Esso riguarda la gestione dei cosiddetti esami di laboratorio e l' $i$ maging clinico in generale. Dovremmo abituarci a un'idea diversa dell'attività clinica. In un futuro prossimo sarà una Al che esaminerà i risultati degli esami e ci indicherà qual è la loro interpretazione suggerendo un'ipotesi diagnostica, prima del passo successivo che sarà quello di consigliarci quali esami sia necessario eseguire per confermare l'ipotesi diagnostica che lei stessa ha formulato. II passo verso una automazione dell'imaging clinico è quasi pronto. Un'azienda tecnologica israeliana la Zebra Medical Vision ha milioni 
di record di immagini delle più varie patologie, a vario stadio e dei più svariati organi. Ha messo a punto un software che mette a confronto le immagini del paziente con l'immenso big data che ha accumulato. In pochi minuti sarà possibile trovare un'immagine uguale a quella del nostro paziente a cui corrisponde una diagnosi certificata. Sarà come viene fatto quando la polizia inserisce nei propri database le impronte digitali di uno sconosciuto e queste vengono confrontate fino a trovarne una alla quale corrisponde una persona.

Ma dobbiamo anche considerare che questo progresso tecnologico si affianca, supporta un nuovo modo di intendere le scienze mediche e cioè quello della medicina cosiddetta personalizzata o di precisione. Essa fa sempre più ricorso a marker biologici e genetici nella scelta delle cure per ottenere efficacia massima con minimi effetti indesiderati.

La principale sfida per la medicina personalizzata comporterà che la gestione di una complessità crescente non potrà che essere decodificata da potenti strumenti di calcolo, da strumenti decisionali nei quali la Al avrà un ruolo centrale.

Bisogna immaginare che saranno le Machine Learning ad analizzare questa mole e questa complessità di dati che le analisi sempre più sofisticate mettono a disposizione dei medici e degli scienziati. Esse saranno capaci di elaborare la grande massa di letteratura scientifica disponibile su una determinata malattia o su un intervento terapeutico e aiutare i medici e i ricercatori in modo critico a fare ipotesi di diagnosi e cura.

\section{Quale sarà dunque il rapporto fra $\mathrm{Al}$, medici e pazienti?}

Intanto dobbiamo aver presente che a causa della nostra alta capacità di adattamento potremmo finire per affidare decisioni cliniche importanti a sistemi automatici incapaci di pensare o di capire poco anche se li chiamiamo Al. Sarà imprescindibile, cruciale avere l'assicurazione, la certezza che il disegno, il controllo e la responsabilità dei sistemi digitali e di tutto ciò che noi affideremmo loro restino fermamente nell'ambito delle decisioni umane. La nuova legislazione europea sul General Data Protection Regulation (15), discussa in questi mesi, dovrebbe perciò garantire, a chiunque sia soggetto a decisioni personali prese in modo del tutto automatico da sistemi artificiali sulla base di algoritmi, il diritto ad avere una spiegazione sulle decisioni prese sulla sua persona. Ecco perché l'uomo resterà insostituibile. È vero, i pazienti molto spesso chiedono di avere a disposizione tecnologie sempre più sofisticate per essere curati meglio. Ma il vero bisogno dei pazienti è di non essere abbandonati e lasciati soli nei momenti difficili della loro vita quando le macchine fanno una diagnosi corretta, asettica, essi hanno bisogno di aver vicino un uomo come loro, un medico che conosce le debolezze dell'anima umana, ne sa comprendere il bisogno ed è pronto a indicare la strada da percorrere, accompagnandoli.
Perché i computer in generale saranno capaci di riconoscere una mela come, e meglio, di noi umani, ma non sanno immaginarne il profumo, il gusto, tantomeno avranno il desiderio di assaggiarla. Manca loro l'essenza della vita, la consapevolezza che caratterizza noi umani. E ancora, il computer Watson sarà capace di fare diagnosi straordinarie, sempre più precise, ma non avrà mai la consapevolezza che dietro una diagnosi esatta ci sono sempre l'ansia di un uomo che teme per la sua vita, il dolore che la malattia porta nell'animo dell'uomo, la sofferenza per il proprio futuro.

Molte professioni saranno eliminate dalle macchine (16), forse quasi sicuramente quelle del medico così come lo intendiamo attualmente. Molto di meno saranno sostituiti gli infermieri che dovranno unire capacità manuali ma non standardizzate ed empatia. Sulla empatia si giocherà il futuro delle professioni. Chi saprà essere empatico, chi avrà le capacità comunicative tali da poter coinvolgere il paziente (17) non solo avrà migliori risultati ma conserverà il suo lavoro, e non è poco.

Non dobbiamo, quindi, aver paura. La differenza fra noi e le macchine è che esse sono insuperabili nel calcolo verticale, lineare; invece il nostro cervello, per quanto a confronto enormemente più lento, è insuperabile perché al contrario del computer è capace di passare in un baleno da una visione verticale a quella orizzontale capace perciò di aggregare concetti non lineari. Ma per affrontare questa sfida serve un nuovo Umanesimo che rimetta al centro del nostro mondo l'uomo, il medico e le sue capacità non solo tecniche ma comunicative, di empowerment, di coinvolgimento tali da portare alla troppo citata ma spesso mai ottenuta alleanza terapeutica.

\section{Disclosures}

Financial support: No financial support was received for this submission

Conflict of interest: The authors have no conflict of interest.

\section{Bibliografia}

1. Clarke AC. 2001: Odissea nello spazio. Traduzione di Bruno Oddera, Milano: Longanesi 1969.

2. Kubrick S. 2001: Odissea nello spazio. Metro-Goldwyn-Mayer (MGM), USA, Gran Bretagna, 1968.

3. Dr. David G. Stork. Dawn of HAL: History of Artificial Intelligence - Dr. Arthur C. Clarke Interview, in 2001: HAL's Legacy Web site, PBS.

4. Cervini M. La terza via al problem solving: prassi euristica e illusione algoritmica, un compromesso. Roma: M.C.A. 1992.

5. Cecile De Jesus. Ross: A Very Smart Artificial Co-worker, Futurism. 11 maggio 2016.

6. High, Rob. The era of cognitive systems: An inside look at IBM Watson and how it works. IBM Corporation, Redbooks, 2012.

7. JPMorgan Chase \& Co., Annual Report 2016.

8. Nilsson NJ. Learning machines. New York: Mcgraw-Hill 1965.

9. LeCun $Y$, Bengio $Y$, Hinton G. Deep learning. Nature. 
2015;521:436-44.

10. Revell T. Al takes on top poker players. New Scientist. 2017; 233:8.

11. Moravčík M, Schmid M, Burch N, et al. DeepStack: Expert-level artificial intelligence in heads-up no-limit poker. Science. 2015; 356(6337):508-13.

12. Bowling $\mathrm{M}$, Burch $\mathrm{N}$, Johanson $\mathrm{M}$, Tammelin O. Heads-up limit hold'em poker is solved. Science. 2015;347(6218): 145-9.

13. Zauderer MG, Gucalp A, Epstein AS, et al. Piloting IBM Watson Oncology within Memorial Sloan Kettering's regional network.
J Clin Oncol. 2014;32:15 (Suppl), abstr e17653.

14. Intelligenza artificiale, "Watson" di IBM insegna in ospedale. La Repubblica, 14 Novembre 2016.

15. Blackmer WS. GDPR: Getting Ready for the New EU General Data Protection Regulation; su: Information Law Group, InfoLowGroup LLP, 5 May 2016.

16. Allarme Onu: i robot sostituiranno il $66 \%$ del lavoro umano. II Sole 24 ore, 18 Novembre 2016.

17. Graffigna G. Verso un paziente 3.0: il Patient Engagement come chiave per un reale protagonismo della persona in sanità. II Sole 24 ore - Sanità, 16 giugno 2017. 